\title{
Cellular heterogeneity-adjusted clonal methylation (CHALM) provides better prediction of gene expression
}

Jianfeng $\mathrm{Xu}^{2 \#}$, Jiejun Shi ${ }^{1,2 \#}$, Xiaodong Cui ${ }^{2}$, Ya Cui1 ${ }^{1,2}$, Jingyi Jessica $\mathrm{Li}^{3}$, Ajay Goel ${ }^{4}, \mathrm{Xi}$

Chen ${ }^{2}$, Jianzhong $\mathrm{Su}^{2 \star}$, Jean-Pierre Issa ${ }^{5 *}$, Wei $\mathrm{Li}^{1{ }^{12 *}}$

\section{Affiliations:}

1Department of Biological Chemistry, School of Medicine, University of California, Irvine, Irvine, CA 92697, USA

${ }^{2}$ Department of Molecular and Cellular Biology, Baylor College of Medicine, Houston, TX 77030, USA.

3Department of Statistics, University of California, Los Angeles, CA 90095, USA.

${ }^{4}$ Department of Molecular Diagnostics and Experimental Therapeutics, Beckman Research Institute of City of Hope, CA 91010, USA.

5The Coriell Institute for Medical Research. Camden, NJ 08103, USA.

"co-first authors

*co-senior authors; Correspondence should be addressed to W.L. (wei.li@uci.edu), J.P.I. (jpissa@coriell.org) or J. S. (sujz@wmu.edu.cn) 


\section{Abstract}

Promoter DNA methylation is a well-established mechanism of transcription repression, but its global correlation with gene expression is surprisingly weak. This weak correlation is due in part to the failure of current methylation quantification methods to consider the heterogeneity among sequenced bulk cells. Here, we introduce $\underline{\text { Cell }}$ Heterogeneity- $\underline{\text { Adjusted }}$ CLonal Methylation (CHALM) as a novel methylation quantification method. CHALM better explains the functional consequences of DNA methylation, such as its correlations with gene expression and H3K4me3. When applied to different methylation datasets, the CHALM method has uniquely detected differentially methylated genes that exhibit distinct biological functions supporting underlying mechanisms. 


\section{Main text}

DNA methylation is an essential epigenetic modification, and its role in transcription repression has been widely studied for decades. Jones et al. (1998) demonstrated that methylated CpGs (mCpGs) in the promoter region are recognized by methyl-CpG-binding domain (MBD) proteins, which subsequently recruit histone deacetylase complexes to repress downstream gene expression ${ }^{1}$. Paradoxically, almost all studies conducted using genome-wide methylation profiling technologies such as whole-genome bisulfite sequencing (WGBS) have demonstrated a poor global correlation between promoter methylation and gene expression ${ }^{2-5}$. For example, Booth et al. ${ }^{2}$ found only slightly negative correlations between transcription and both $5 \mathrm{mC}$ and $5 \mathrm{hmC}$ levels in promoter $\mathrm{CpG}$ islands (CGIs), whose role in transcription regulation has been well-established ${ }^{6}$. Efforts to address this paradox have shown that complex methylation patterns of regions much longer than promoters ${ }^{7,8}$ (e.g., a 10-kb window surrounding the transcription start site) better explain gene expression. However, why promoter methylation alone is only weakly correlated with gene expression has not been directly addressed.

Here, we show that the poor correlation between promoter methylation and gene expression is due in part to the overly simplistic nature of the traditional DNA methylation quantification method (i.e., it determines just the mean methylation level of every $\mathrm{CpG}$ within a promoter) ${ }^{9}$. A key disadvantage of this traditional method is that it fails to account for heterogeneity among sequenced bulk cells but treats CpGs within or across cells as if they are identical (Supplementary Fig. 1a). For example, $20 \%$ of the cells in population A (Fig. 1a) are fully methylated in a promoter region, whereas the rest of the cells are fully unmethylated. In cell population B (Fig. 1b), there is one $\mathrm{mCpG}$ site per promoter in every cell. The traditional quantification method would indicate that the methylation level of this promoter is the same in both populations. Nevertheless, as previous studies demonstrated that a single $\mathrm{mCpG}$ is 
sufficient for recruiting MBD proteins ${ }^{10,11}$ for gene repression, we hypothesized that this promoter would be repressed in $20 \%$ and $100 \%$ of cells in these two populations, respectively. Apparently, the traditional method fails to capture the potential expression difference. To avoid this pitfall, we developed a novel methylation quantification method: Cell Heterogeneity-Adjusted cLonal Methylation (CHALM), which leverages the fact that each bisulfite sequencing read likely represents a single cell within the sequenced bulk cells. Instead of calculating the mean methylation level of all CpG sites, CHALM quantifies the promoter methylation as the ratio of methylated reads (with $\geq 1 \mathrm{mCpG}$ ) to total reads mapped to a given promoter region. According to CHALM, the promoter methylation levels of these two cell populations would be 0.2 and 1 , which might better explain the transcription activity. As expected, on promoter CGls, CHALM-determined methylation levels fit a bimodal distribution (Supplementary Fig. 1b) and are usually higher than traditionally determined methylation levels (Fig. 1c).

For methods comparison, we mainly focus on promoter CGls, which have been extensively studied for the relationship between DNA methylation and gene expression. We first assessed the power of the CHALM method in terms of predicting gene expression on a genome-wide scale using a CD3 primary cell dataset. Although the methylation levels calculated by both CHALM and traditional methods were anti-correlated with gene expression (Fig. 2a; Supplementary Fig. 2a, 3a, 3c and 4), the CHALM-determined methylation levels exhibited a more linear and monotonic relationship with gene expression. As expected, lowly methylated promoter CGls exhibited a very weak correlation between traditional methylation and gene expression ${ }^{12-14}$ (Fig. 2b; Supplementary Fig. 2b and 4). Surprisingly, we observed a much stronger correlation between gene expression and CHALM-determined methylation (Fig. 2b; Supplementary Fig. 2c and 4). In addition, although we primarily focused on promoter CGIs (Methods), CHALM also outperformed the 
traditional methods in several other widely studied genomic regions (Supplementary Fig. 5-7).

DNA methylation is also known to be mutually exclusive with $\mathrm{H} 3 \mathrm{~K} 4 \mathrm{me}$, which is strongly associated with gene expression. Unmethylated H3K4 is capable of releasing the autoinhibition of DNMT3A by disrupting the interaction between the ATRX-DNMT3-DNMT3L and catalytic domains, thereby inducing de novo methylation ${ }^{15,16}$. We therefore examined the relationship between DNA methylation and H3K4me3 level in promoter CGIs. With both the traditional and CHALM methods, we consistently observed a negative Spearman correlation between methylation level and H3K4me3 (Fig. 2c and Supplementary Fig. 3b, 3d and 8). However, when we focused on genes with low methylation levels, only CHALM-determined methylation was significantly anti-correlated with H3K4me3 level (Fig. 2d and

Supplementary Fig. 8), suggesting that the CHALM method provides a better representation of the mutually exclusive relationship between DNA methylation and H3K4me3.

To further illustrate that CHALM better explains transcription activity, we next examined in detail two genes with similar methylation levels as determined by the traditional method but with different transcription activities. We found that most reads mapped to the promoter CGI of HIST2H2BF were fully unmethylated (low CHALM-determined methylation level), which explained the high transcription activity (Fig. 2e). A large fraction of reads of the repressed gene SSTR5 had at least one $\mathrm{mCpG}$, which indicated that the high methylation level as determined by the CHALM method was responsible for transcription repression (Fig. 2f). Collectively, these results demonstrate that the CHALM method provides better prediction of gene expression. 
Since the CHALM method quantifies the ratio of methylated reads, its performance depends on the definition of methylated reads, i.e. reads with at least $\mathrm{N} \mathrm{mCpG}$ sites. We evaluated CHALM based on varying definitions of methylated reads and found that CHALM performed the best when $\mathrm{N}$ equals to 1 (Supplementary Fig. 9). In addition, we noticed that CHALM requires an average $\mathrm{CpG}$ depth of more than $7 x$ in order to achieve the optimal performance (Supplementary Fig. 10). Furthermore, we found that read length would also influence the CHALM performance. For WGBS datasets, the read length is typically around $100 \mathrm{bp}$, which is too short to cover the promoter CGI region, thus potentially adversely impacting the CHALM performance (Supplementary Fig. 11). We therefore employed an SVD-based imputation method ${ }^{17-19}$ to extend the reads. After method validation, we extended the reads to different lengths and observed that the CHALM performance improved, approaching a plateau at a read length of $300 \mathrm{bp}$ (Supplementary Fig. 12-14). Given these results, CHALM prefers paired-end sequencing data, as the effective read length is twice that of single-end sequencing data (Supplementary Fig. 15 and 16).

A number of factors must be taken into account when evaluating the power of DNA methylation data for predicting gene expression, such as the distance between the read and the transcription start site and the weight of reads with more than one $\mathrm{mCpG}$. Therefore, in order to maximize the amount of useful information extracted from high-throughput sequencing data, we processed the raw sequencing data into an image-like data structure in which one channel contained methylation information and the other contained read location information (Supplementary Fig. 17). These data are then further processed using a convolutional deep neural network for gene expression prediction. As expected, this deeplearning model outperformed a linear model trained using either traditionally determined or CHALM-determined methylation levels (Fig. 3a; Supplementary Fig. 18a). Notably, the performance of this deep-learning model was markedly compromised after we shuffled the 
$\mathrm{mCpG}$ position (assigning $\mathrm{mCpG}$ to random reads) while keeping the total number of mCpGs unchanged but entirely disrupting the clonal information (Fig. 3b and 3c;

Supplementary Fig. 18-20). This result demonstrates the crucial role of clonal information in predicting gene expression. We also demonstrated that this deep-learning prediction model outperforms a previously published method ${ }^{8}$ in terms of predicting gene expression based on promoter CGI methylation levels (Supplementary Fig. 21). Finally, it is worth noting that the predicted values output by the deep-learning model should not be used as methylation levels, despite that they have a higher correlation with gene expression than the CHALM-determined methylation levels do. The reason is that the deep-learning model is trained to predict gene expression, and thus its output predicted values rely on not only methylation data but also gene expression data; also, the predicted values are derived only for the prediction purpose but ignore other important biological aspects of DNA methylation.

To demonstrate the utility of the CHALM method, we compared it to the traditional method for identifying differentially methylated genes with promoter CGls in paired cancerous and normal lung tissue samples ${ }^{20}$. The correlation between differential methylation and differential gene expression was significantly greater when the methylation level was calculated using the CHALM method (Fig. 4a). In addition, the CHALM method not only recovered most of the traditional method-identified hypermethylated genes but also identified a subset of genes that are overlooked by the traditional method. Consistent with studies showing that Polycomb-mediated H3K27me3 pre-marks gene promoters for de novo methylation during tumorigenesis ${ }^{21-23}$, the hypermethylated genes identified by both methods were highly enriched with $\mathrm{H} 3 \mathrm{~K} 27 \mathrm{me} 3$ in normal lung tissue. Interestingly, the CHALM-unique hypermethylated genes were more enriched in $\mathrm{H} 3 \mathrm{~K} 27$ me3 than hypermethylated genes uniquely determined by the traditional method, suggesting that CHALM provides more accurate identification of hypermethylated promoter CGls (Fig. 4b; Supplementary Fig. 22 
and 23). Furthermore, with reads extended by imputation, the CHALM method identifies more hypermethylated promoter CGls that are also enriched with H3K27me3

\section{(Supplementary Fig. 24).}

We also demonstrated the utility of the CHALM method for calling de novo differentially methylated regions (DMRs) ${ }^{24}$. Lung adenocarcinoma (LUAD) is a slow-growing non-small cell lung cancer that accounts for around $40 \%$ of lung cancers ${ }^{25}$. Upon treatment with tyrosine kinase inhibitors, a subset of resistant LUADs transform into small cell lung cancer (SCLC), a more-aggressive neuroendocrine tumor ${ }^{26-28}$. To delineate the epigenetic 'rewiring' that underlies this transformation, we called de novo DMRs between LUAD and SCLC using both the traditional and CHALM methods. We found that a larger fraction of the CHALM unique DMRs were annotated to gene promoter regions (Supplementary Fig. 25). In addition, CHALM-determined hypomethylated DMRs in SCLC were more highly enriched in genes of the neuroactive ligand-receptor interaction pathway, which is reportedly activated in $\operatorname{SCLC}^{29}$ (Fig. 5a). Expression of genes from this pathway with hypomethylated DMRs was consistently up-regulated in SCLC (Fig. 5b). In addition, a drug repositioning study reported that potential drugs for treating SCLC are enriched in targeting genes associated with the neuroactive ligand-receptor interaction pathway, indicating that this pathway plays a crucial role in SCLC ${ }^{30}$. Collectively, CHALM data suggest that DNA hypomethylation is involved in activating the neuroactive ligand-receptor interaction pathway during the development of SCLC.

Somatostatin receptors (SSTRs) are G-protein-coupled receptors in the neuroactive ligandreceptor interaction pathway that mediate somatostatin's inhibition of cell proliferation, endocrine signaling, and neurotransmission ${ }^{31}$. Given their high expression in neuroendocrine tumors, SSTRs (along with other marker genes) have been used for the detection of 
neuroendocrine tumors ${ }^{32,33}$. Several somatostatin analogs, including octreotide (SMS 201995) and vapreotide (RC-160), have been proposed for use in treating neuroendocrine tumors, including SCLC ${ }^{34}$. Consistent with the up-regulation of SSTR expression in SCLC (Fig. 5c and Supplementary Fig. 26), we also observed significant hypomethylation of the promoter regions of SSTR1, SSTR2, and SSTR5 in SCLC, but only when using the CHALM method (Fig. 5d and Supplementary Fig. 27).

To demonstrate the robustness of the CHALM method, we also used it to identify de novo DMRs during the aging process (mice) and during the development of Alzheimer's disease (humans). In both scenarios, CHALM identified more DMRs closely related to the underlying biological mechanisms (Supplementary Fig. 28 and 29).

We would like to reiterate that CHALM is a method for quantifying cell heterogeneityadjusted mean methylation, but it is not a method for quantifying methylation heterogeneity per se. Therefore, CHALM is fundamentally different from all of the epigenetic heterogeneity and entropy methods reported before, such as $\mathrm{PDR}^{35}$, epipolymorphism ${ }^{36}$ and Shannon entropy ${ }^{37}$. We compared CHALM and these three heterogeneity methods to the traditional methylation method and noted that CHALM exhibited the best correlation with the traditional methylation method. In contrast, the three above-mentioned heterogeneity metrics fit a bellshaped curve with traditional methylation and thus are not appropriate for direct quantification of methylation, as they cannot distinguish CGls with low methylation levels (i.e., 0.0-0.2) from those with high methylation levels (i.e., 0.8-1.0) (Supplementary Fig. 30-33).

In conclusion, our data indicate that the novel CHALM method, which incorporates cell heterogeneity information into DNA methylation quantification, provides a better explanation 
for the functional consequences of DNA methylation, as evidenced by the demonstrated correlation with gene expression and $\mathrm{H} 3 \mathrm{~K} 4 \mathrm{me} 3$. We realize that DNA methylation in the promoter region and gene body exhibit different relationships with transcription activity. However, as a causal relationship between gene body methylation and gene expression has not been clearly established 6 , we primarily focused on the promoter regions. We further illustrated the importance of clonal information in quantifying DNA methylation using a deep learning model and demonstrated the advantages of the CHALM method for more accurate identification of functionally related DMRs. Finally, although the definition of CHALM involves the ratio of methylated reads, CHALM is actually intended for quantification of the adjusted methylation level for each CpG site, which makes our method compatible with most existing downstream analysis tools, such as differentially methylated cytosine or DMR calling tools (Supplementary Fig. 34). It is anticipated that the CHALM method will be of great value for research that aims to fully delineate the role of DNA methylation in transcription regulation. The CHALM method is available at https://github.com/JianfengXu93/CHALM.

\section{Methods}

\section{RNA-seq analysis}

Raw sequencing data of CD3 primary cells (GSM1220574), CD14 primary cells (GSM1220575), cancerous and normal lung tissue (GSE70091) and small cell lung cancer (SCLC, GSE60052) were downloaded from Gene Expression Omnibus (GEO). Raw sequencing data of lung adenocarcinoma (LUAD) samples were downloaded from GDC legacy archive. We used Trimmomatic $(0.35)^{38}$ to trim low-quality bases and sequencing adapters. TopHat (2.1.0) $)^{39}$ was then used to align sequencing reads to the hg19 human reference genome with default parameters. The hg19 GTF annotation file for transcriptome alignment was downloaded from UCSC annotation database. We used Cufflinks (2.2.1 $)^{40}$ to calculate Fragments Per Kilobase of transcript per Million mapped reads (FPKM) for 
annotated transcripts. As for differential expression analysis, read counts of transcripts were first calculated by HTSeq (htseq-count, 2.7) ${ }^{41}$. DEseq2 $(1.20)^{42}$ was then used to calculate the expression difference and the statistical importance.

\section{WGBS data pre-processing}

Raw bisulfite sequencing data of CD3 primary cells (GSM1186660), CD4 primary cells (GSM1186661), cancerous and normal lung tissue (GSE70091), LUAD and SCLC (GSE52271) were downloaded from GEO. After trimming low-quality bases and sequencing adapters, we used BSMAP $(2.90)^{43}$ to align reads to hg19 human reference genome with default parameters. The methratio.py (from BSMAP package) script was then used to calculate the methylation ratios of $\mathrm{CpG}$ sites. Only CpG sites covered by at least 4 reads are retained for the downstream analyses.

\section{Promoter CpG islands (CGIs)}

Annotation files for gene position and $\mathrm{CpG}$ islands for hg19 assembly were downloaded from UCSC table browser. Promoter CGls are defined as CGls exhibiting overlap with 2-kb windows centered on gene transcription start sites.

\section{Quantifying the methylation levels of promoter CGIs}

The aforementioned traditional method for calculating promoter methylation level mainly refers to the "mean methylation level", which is computed as

$$
1 / n \sum_{i=1}^{n} C_{i} /\left(C_{i}+T_{i}\right)
$$

where $C_{i}, T_{i}$ are the counts of methylated cytosine and unmethylated cytosine on the CpG $i$ of the promoter, respectively.

In our work, we also discussed another traditional method, i.e. "weighted methylated level", which is computed as 


$$
\sum_{i=1}^{n} C_{i} / \sum_{i=1}^{n} C_{i}+T_{i}
$$

where $C_{i}, T_{i}$ are the counts of methylated cytosine and unmethylated cytosine on the $\mathrm{CpG} i$ of the promoter, respectively.

The "CHALM methylation level" is computed as

$$
n_{m} /\left(n_{m}+n_{u}\right)
$$

where $n_{m}, n_{u}$ are the counts of methylated reads and unmethylated reads mapped to the promoter regions, respectively. Reads with at least one $\mathrm{mCpG}$ site are defined as methylated reads.

\section{Differentially methylated regions (pre-defined regions)}

For traditional method, differential methylation of promoter CGIs were calculated by Metilene ('pre-defined regions' mode, 0.2-7) with default parameters.

For CHALM, differential methylation of promoter CGIs were calculated based on betabinomial model. For a promoter CGI $i$, we denoted the counts of methylated reads, the counts of unmethylated reads and CHALM methylation ratio as $n_{m i}, n_{u i}, p_{i}$, respectively. The $n_{m i}$ and $n_{u i}$ are observed values while $p_{i}$ is unknown. Given that sequenced reads are sampled from the sequencing cell population, we used binomial distribution to model the methylated reads

$$
n_{m i} \sim B\left(n_{m i}+n_{u i}, p_{i}\right)
$$

where the $p_{i}$ follows a beta distribution beta $\left(\alpha_{i}, \beta_{i}\right)$, which can be estimated by empirical Bayes method. Similar method has already been implemented in our previously published MOABS package ${ }^{44}$. We then repurposed MOABS to calculate the differential CHALM methylation. The cutoff for significant differential methylation: absolute methylation changes are equal to or more than 0.1 and FDR adjusted $p$-value is less than 0.05 . 


\section{Differentially methylated regions (de novo)}

For traditional method, de novo DMRs are identified by Metilene ('de novo' mode, $0.2-7$ ) with default parameters.

For CHALM, we first calculated the CHALM methylation ratio for each CpG site. After reads alignment, we scaned each read for $\mathrm{mCpG}$. If a read had at least one $\mathrm{mCpG}$, other $\mathrm{CpG}$ sites on the same read would be treated as $\mathrm{mCpG}$ as well. Then, the CHALM methylation ratio would be calculated with the methratio.py script from BSMAP. CpG sites covered by at least 4 reads were selected for calling de novo DMRs by Metilene ('de novo' mode).

Identified de novo DMRs by both traditional method and CHALM were annotated to the nearest gene. We then performed pathway enrichment and gene ontology analysis for the differentially methylated genes by using DAVID (6.8) and Enrichr.

\section{ChIP-seq data analysis}

H3K4me3 ChIP-seq datasets for CD3 primary cell, CD14 primary cell were downloaded from Roadmap project. Sequencing reads were aligned to hg19 human reference by bowtie2 (2.2.7, local mode). We then counted mapped reads for each promoter CGI by htseq-count with default setting. Finally, the H3K4me3 ChIP-seq signal intensity of a promoter CGI was defined as read counts normalized by the length of the promoter CGI.

\section{Balance the promoter CGIs set}

Since most promoter CGls are unmethylated, the distribution of methylation value of promoter CGIs is severely biased to 0 . In order to balance the distribution, all promoter CGIs (around 12000) were split into 200 bins based on their traditional methylation value. For 
each bin, up to 60 promoter CGls were randomly selected. The final CGIs set (around 3000 promoter CGIs) is composed of the selected promoter CGls from 200 bins.

\section{Permutation test to assess the statistical significance of the difference between two}

\section{Spearman correlation coefficients}

Two samples, which have the same size and are used to calculate two Spearman correlation coefficients, $r_{1}$ and $r_{2}$, are first pooled into a single sample. In the $b$-th permutation run, we randomly divided this pooled sample into two halves, which would be used to compute two permutated Spearman correlation coefficients, $r_{1}^{(b)}$ and $r_{2}^{(b)}$. Then we calculated the difference $r_{d}^{(b)}=r_{2}^{(b)}-r_{1}^{(b)}$. We performed 10,000 independent permutation runs to obtain 10,000 differences under the null hypothesis that the two samples are from the same distribution: $r_{d}^{(1)}, \ldots, r_{d}^{(B)}$. Finally, we compared the original difference $r_{d}=r_{2}-r_{1}$ to these 10,000 differences to compute a p-value defined as $\frac{1}{B} \sum_{b=1}^{B} I\left(r_{d}^{(b)} \geq r_{d}\right)$ for a one-sided test.

\section{Missing value imputation}

Since the length of most public bisulfite sequencing datasets is around 100bp while the length of promoter CGIs ranges from $201 \mathrm{bp}$ to several $\mathrm{kb}$, a single read can only capture a small proportion of $\mathrm{CpG}$ sites of a promoter $\mathrm{CGI}$. In order to rescue the information from the uncaptured CpG sites, low-rank SVD approximation (estimated by the EM algorithm) was used to extend the read based on the information of nearby reads ${ }^{17}$. Promoter CGls larger than $500 \mathrm{bp}$ and with more than 300 mapped reads were selected for imputation. Mapped reads of a promoter CGI were converted into a matrix with column representing $\mathrm{CpG}$ sites of this promoter CGI and row representing different reads. Each row contained the methylation status (mCpG: 1; CpG: 0) of CpG sites captured by a single read. The methylation status of 
the CpG site uncaptured by reads was label as NA and will be imputed by the 'impute.svd' function from bcv package ${ }^{17,18}$.

\section{Deep learning prediction}

Promoter CGIs with more than 50 mapped reads were selected for deep learning prediction. The methylation status (mCpG: 1; CpG: 0 ) and the distance of mapped reads to the TSS would be stored into a 3D array. The $3 D$ array is similar to the data structure for storing the positions and pixel information of an image. The first dimension is for storing the mapped reads, which was sorted by the read's methylation fraction

$$
f_{m}=N_{m} /\left(N_{m}+N_{u}\right)
$$

where $N_{m}, N_{u}$ refers to the number of methylated CpG and unmethylated CpG on this read, respectively. The length of this dimension is 200 . When there were less than 200 mapped reads $\left(N_{r}<200\right)$, pseudo-reads were generated by bootstrapping from actual reads. When there were more than 200 mapped reads $\left(N_{r}>200\right), 200^{\star} F_{\text {size }}\left(N_{r}-200<200^{\star} F_{\text {size }}<N_{r}\right)$ reads were randomly selected. Selected reads were then sorted based on methylation fraction and split into 200 bins, with $F_{\text {size }}$ reads in each bin. Finally, a pseudo-read was generated based on the mean value of each bin. $N_{r}$ and $F_{\text {size }}$ refer to the number of mapped reads and the size factor, respectively.

The second dimension is to store the methylation status of the $\mathrm{CpG}$ sites on the reads. The dimension length is 10 , which stores the methylation status of $10 \mathrm{CpG}$ sites from a sequencing read. When there were less than $10 \mathrm{CpG}$ sites, the methylation status of a read CpG site was expanded to a pseudo-CpG site. When there were more than $10 \mathrm{CpG}$ sites, the methylation levels of adjacent CpG sites were merged (Supplementary Fig. 35). 
The last dimension contains two channels: one channel storing methylation information and the other one storing the distance of mapped reads to TSS.

To train this image-like 3D array $(200 \times 10 \times 2)$ data, we built a CNN model with PyTorch (version 1.2). Specifically, the input layer is attached to three sequential Conv2d layers along with RELU activation function. The kernel size of the three Conv2d layers is $(5,1),(4,1)$ and $(3,1)$ respectively. The stride for all Con2d layers is $(1,1)$. Since the second dimension of the input data is small, we did not include pooling layer in our model. The final output layer of this CNN model is a linear regression layer. And in order to prevent overfitting, a dropout layer $(p=0.2)$ was added between the convolution layer and the fully connected layer. We then trained the CNN model using Adam as optimizer and MSELoss as loss function in batches of 32 promoter CGls.

In order to disrupt the clonal information in the control group, we randomly assigned the mCpGs to mapped reads but kept the total number of mCpGs unchanged. We then sorted the reads based on the methylation fraction to obtain the input matrix, which was used for prediction.

Since most promoter CGls are unmethylated, the original dataset was downsampled to generate a relatively evenly-distributed dataset (balanced promoter CGI set). Downsampled datasets were then randomly split into training set and test set in a manner of 50\%-50\%. After converting the raw bisulfite sequencing reads into the aforementioned 3D matrix, we trained a convolutional neural network (CNN) model to predict gene expression based on this matrix (Supplementary Fig. 8). The testing set was then used to evaluate the performance of this model. 
As a contribution to the community, we also generated a pretrained CNN model by using the RNA-seq and WGBS datasets of 23 different normal tissues from the Roadmap epigenomic project. This pretrained model is ready to use for studying the relationship between DNA methylation and gene expression in other datasets that are of researchers' interest.

\section{Acknowledgements}

We acknowledge J. Rosen, H. Liang, L. Shen, N. Weigel and D. Lamb for constructive discussions and support. This work is funded by the NIH R01HG007538.

\section{Author contributions}

J.X., J.P.I., and W.L. conceived and developed the outline of this research. J.X. and J.S. wrote the tools and performed data analysis and method evaluations. J.X. and X.C. developed the script for deep learning analysis. A.G., X.C., J.S. and J.L. assisted with the manuscript. J.X., J.S. and W.L. wrote the paper.

\section{Competing interests}

The authors declare no competing financial interests.

\section{References}

1 Jones, P. L. et al. Methylated DNA and MeCP2 recruit histone deacetylase to repress transcription. Nature genetics 19, 187 (1998).

$2 \quad$ Booth, M. J. et al. Quantitative sequencing of 5-methylcytosine and 5hydroxymethylcytosine at single-base resolution. Science 336, 934-937 (2012).

3 Farlik, M. et al. DNA methylation dynamics of human hematopoietic stem cell differentiation. Cell stem cell 19, 808-822 (2016).

4 Ley, T. J. et al. DNMT3A mutations in acute myeloid leukemia. New England Journal of Medicine 363, 2424-2433 (2010).

5 Wagner, J. R. et al. The relationship between DNA methylation, genetic and expression inter-individual variation in untransformed human fibroblasts. Genome biology 15, R37 (2014). 
6 Jones, P. A. Functions of DNA methylation: islands, start sites, gene bodies and beyond. Nat Rev Genet 13, 484-492, doi:10.1038/nrg3230 (2012).

7 Schlosberg, C. E., VanderKraats, N. D. \& Edwards, J. R. Modeling complex patterns of differential DNA methylation that associate with gene expression changes. Nucleic acids research 45, 5100-5111 (2017).

8 Kapourani, C. A. \& Sanguinetti, G. Higher order methylation features for clustering and prediction in epigenomic studies. Bioinformatics 32, i405-i412, doi:10.1093/bioinformatics/btw432 (2016).

9 Schultz, M. D., Schmitz, R. J. \& Ecker, J. R. 'Leveling'the playing field for analyses of single-base resolution DNA methylomes. Trends in Genetics 28, 583-585 (2012).

10 Ohki, I. et al. Solution structure of the methyl-CpG binding domain of human MBD1 in complex with methylated DNA. Cell 105, 487-497 (2001).

11 Lewis, J. D. et al. Purification, sequence, and cellular localization of a novel chromosomal protein that binds to methylated DNA. Cell 69, 905-914 (1992).

12 Bell, J. T. et al. DNA methylation patterns associate with genetic and gene expression variation in HapMap cell lines. Genome Biol 12, R10, doi:10.1186/gb-2011-12-1-r10 (2011).

13 Lou, S. et al. Whole-genome bisulfite sequencing of multiple individuals reveals complementary roles of promoter and gene body methylation in transcriptional regulation. Genome biology 15, 408 (2014).

14 Roadmap Epigenomics, C. et al. Integrative analysis of 111 reference human epigenomes. Nature 518, 317-330, doi:10.1038/nature14248 (2015).

15 Ooi, S. K. et al. DNMT3L connects unmethylated lysine 4 of histone $\mathrm{H} 3$ to de novo methylation of DNA. Nature 448, 714-717, doi:10.1038/nature05987 (2007).

16 Guo, X. et al. Structural insight into autoinhibition and histone H3-induced activation of DNMT3A. Nature 517, 640 (2015).

17 Owen, A. B. \& Perry, P. O. Bi-cross-validation of the SVD and the nonnegative matrix factorization. The annals of applied statistics 3, 564-594 (2009).

18 PO, P. Bcv: cross-Validation for the SVD (bi-cross-validation). $R$ package version 1 (2009).

19 Troyanskaya, O. et al. Missing value estimation methods for DNA microarrays. Bioinformatics 17, 520-525 (2001).

$20 \mathrm{Li}, \mathrm{X}$., Liu, Y., Salz, T., Hansen, K. D. \& Feinberg, A. Whole-genome analysis of the methylome and hydroxymethylome in normal and malignant lung and liver. Genome Res 26, 1730-1741, doi:10.1101/gr.211854.116 (2016).

21 Ohm, J. E. et al. A stem cell-like chromatin pattern may predispose tumor suppressor genes to DNA hypermethylation and heritable silencing. Nature genetics 39, 237 (2007).

22 Schlesinger, Y. et al. Polycomb-mediated methylation on Lys27 of histone H3 premarks genes for de novo methylation in cancer. Nat Genet 39, 232-236, doi:10.1038/ng1950 (2007).

23 Widschwendter, M. et al. Epigenetic stem cell signature in cancer. Nat Genet 39, 157-158, doi:10.1038/ng1941 (2007).

24 Juhling, F. et al. metilene: fast and sensitive calling of differentially methylated regions from bisulfite sequencing data. Genome Res 26, 256-262, doi:10.1101/gr.196394.115 (2016). 
25 Imielinski, M. et al. Mapping the hallmarks of lung adenocarcinoma with massively parallel sequencing. Cell 150, 1107-1120 (2012).

26 Lee, J.-K. et al. Clonal history and genetic predictors of transformation into small-cell carcinomas from lung adenocarcinomas. Journal of Clinical Oncology 35, 3065-3074 (2017).

27 Niederst, M. J. et al. RB loss in resistant EGFR mutant lung adenocarcinomas that transform to small-cell lung cancer. Nature communications 6, 6377 (2015).

28 Oser, M. G., Niederst, M. J., Sequist, L. V. \& Engelman, J. A. Transformation from non-small-cell lung cancer to small-cell lung cancer: molecular drivers and cells of origin. The Lancet Oncology 16, e165-e172 (2015).

29 Ocak, S. et al. DNA copy number aberrations in small-cell lung cancer reveal activation of the focal adhesion pathway. Oncogene 29, 6331 (2010).

30 Jahchan, N. S. et al. A drug repositioning approach identifies tricyclic antidepressants as inhibitors of small cell lung cancer and other neuroendocrine tumors. Cancer discovery 3, 1364-1377 (2013).

31 Weckbecker, G. et al. Opportunities in somatostatin research: biological, chemical and therapeutic aspects. Nature reviews Drug discovery 2, 999 (2003).

32 Modlin, I. M. et al. A multianalyte PCR blood test outperforms single analyte ELISAs (chromogranin A, pancreastatin, neurokinin A) for neuroendocrine tumor detection. Endocrine-related cancer 21, 615-628 (2014).

33 Oberg, K. et al. Consensus on biomarkers for neuroendocrine tumour disease. The Lancet Oncology 16, e435-e446 (2015).

34 O’Byrne, K. J., Schally, A. V., Thomas, A., Carney, D. N. \& Steward, W. P. Somatostatin, its receptors and analogs, in lung cancer. Chemotherapy 47, 78-108 (2001).

35 Landau, D. A. et al. Locally disordered methylation forms the basis of intratumor methylome variation in chronic lymphocytic leukemia. Cancer cell 26, 813-825 (2014).

36 Landan, G. et al. Epigenetic polymorphism and the stochastic formation of differentially methylated regions in normal and cancerous tissues. Nature genetics 44, 1207 (2012).

37 Klughammer, J. et al. The DNA methylation landscape of glioblastoma disease progression shows extensive heterogeneity in time and space. Nature medicine 24, 1611 (2018).

38 Bolger, A. M., Lohse, M. \& Usadel, B. Trimmomatic: a flexible trimmer for Illumina sequence data. Bioinformatics 30, 2114-2120 (2014).

39 Trapnell, C., Pachter, L. \& Salzberg, S. L. TopHat: discovering splice junctions with RNA-Seq. Bioinformatics 25, 1105-1111 (2009).

40 Trapnell, C. et al. Differential gene and transcript expression analysis of RNA-seq experiments with TopHat and Cufflinks. Nature protocols 7, 562 (2012).

41 Anders, S., Pyl, P. T. \& Huber, W. HTSeq-a Python framework to work with highthroughput sequencing data. Bioinformatics 31, 166-169 (2015).

42 Love, M. I., Huber, W. \& Anders, S. Moderated estimation of fold change and dispersion for RNA-seq data with DESeq2. Genome biology 15, 550 (2014).

$43 \mathrm{Xi}, \mathrm{Y}$. \& Li, W. BSMAP: whole genome bisulfite sequence MAPping program. BMC bioinformatics 10, 232 (2009). 
bioRxiv preprint doi: https://doi.org/10.1101/2020.02.23.961813; this version posted February 25, 2020. The copyright holder for this

preprint (which was not certified by peer review) is the author/funder. All rights reserved. No reuse allowed without permission.

44 Sun, D. et al. MOABS: model based analysis of bisulfite sequencing data. Genome biology 15, R38 (2014). 
a

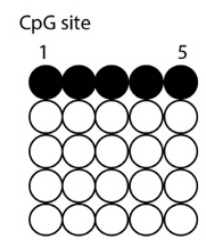

b

Traditional methylation: 0.2

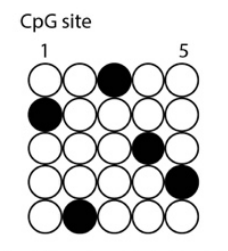

\section{CpG site}

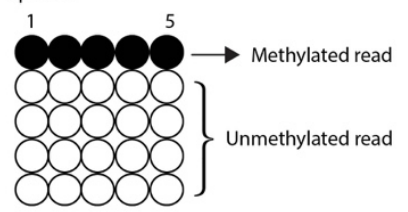

Ratio of methylated reads (CHALM): 0.2

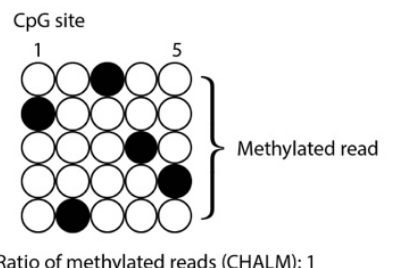

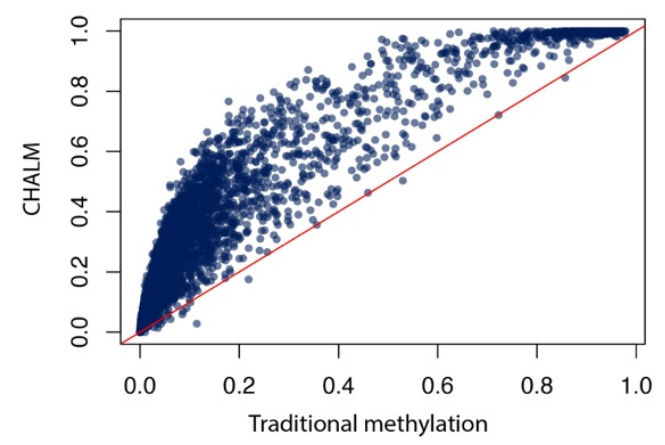

Figure 1. Cell Heterogeneity-Adjusted cLonal Methylation (CHALM) is a novel DNA methylation quantification method adjusted to account for cell heterogeneity. (a) and (b) show two different methylation patterns of a promoter region that cannot be distinguished by the traditional method. (c) Scatter plot shows a comparison of the methylation level calculated by the traditional and CHALM methods for the promoter CGIs of CD3 primary cells. 
a

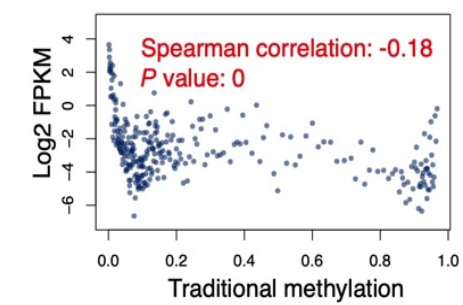

b

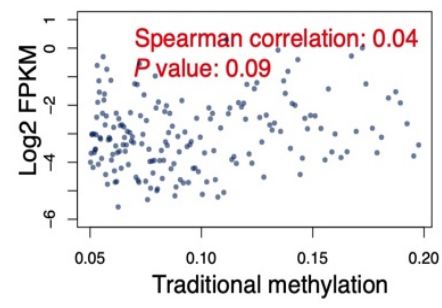

C

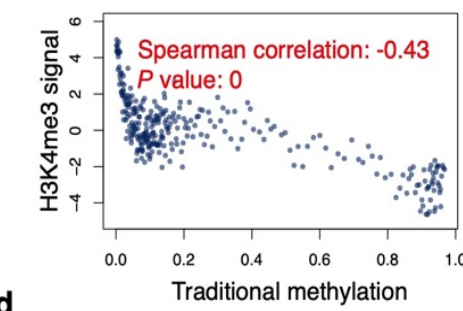

d

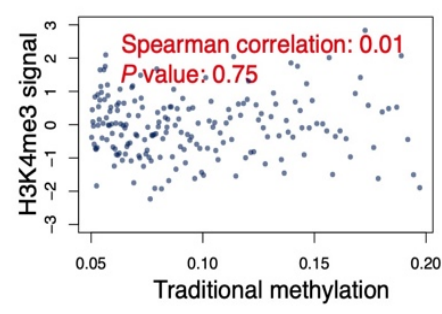

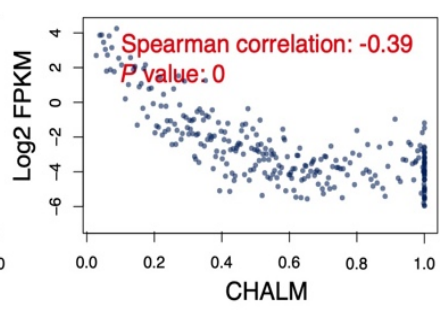
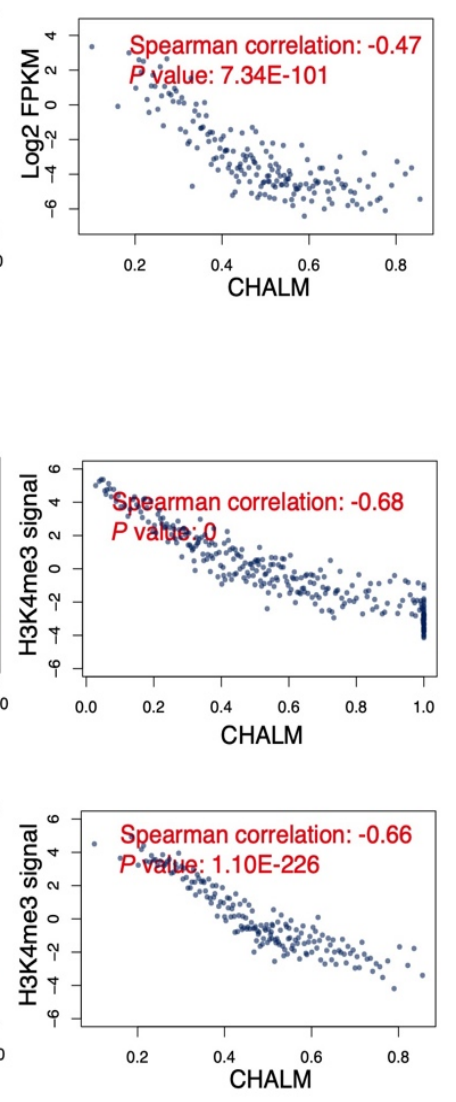

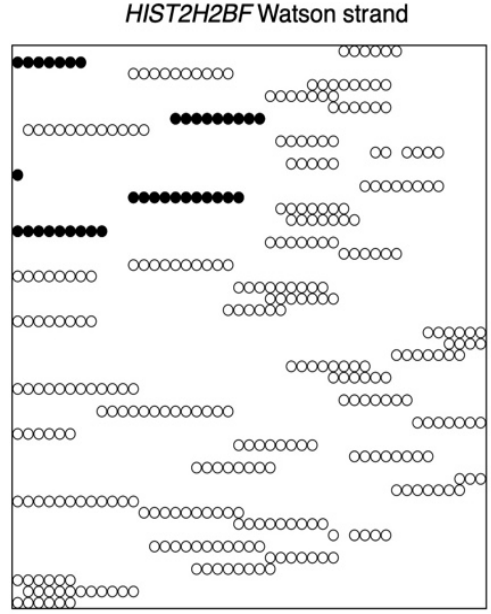

Traditional methylation: 0.10

CHALM: 0.10

FPKM: 77.30

H3K4me3 ChIP-seq signal: 35.02

$\mathbf{f}$

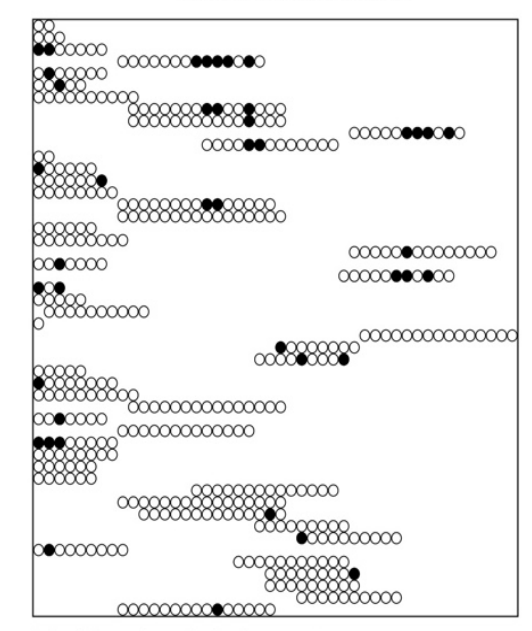

Traditional methylation: 0.09

CHALM: 0.54

FPKM: 0.00

H3K4me3 ChIP-seq signal: 0.00

Figure 2. The CHALM method better predicts gene expression. (a) Scatter plots show the correlation between gene expression and methylation level calculated using both methods. Balanced promoter CGIs (Methods) of CD3 primary cells are used. Each data point represents the average value of 10 promoter CGls, and the Spearman correlation is calculated based on original data for each promoter CGI. Comparison of correlation (between the traditional method and CHALM) $P$ values calculated by permutation (Methods): $<1 \times 10^{-4}$ (b) Similar to (a) but focusing on low-methylation genes. Comparison of correlation permutation $P$ values: $<1 \times 10^{-4}$. (c) Scatter plots show the correlation between H3K4me3 ChIP-seq intensity and methylation level calculated by the traditional and CHALM methods. Balanced promoter CGIs are used. Comparison of correlation permutation $P$ values: $<1 \times 10^{-4}$. (d) Similar to (c) but focusing on low-methylation genes. Comparison of correlation permutation $P$ values: $<1 \times 10^{-4}$. (e) and (f) Methylation status of reads mapped to the promoter CGI of HIST2H2BF or SSTR5, respectively. Black circles: mCpG; white circles: CpG. 
a

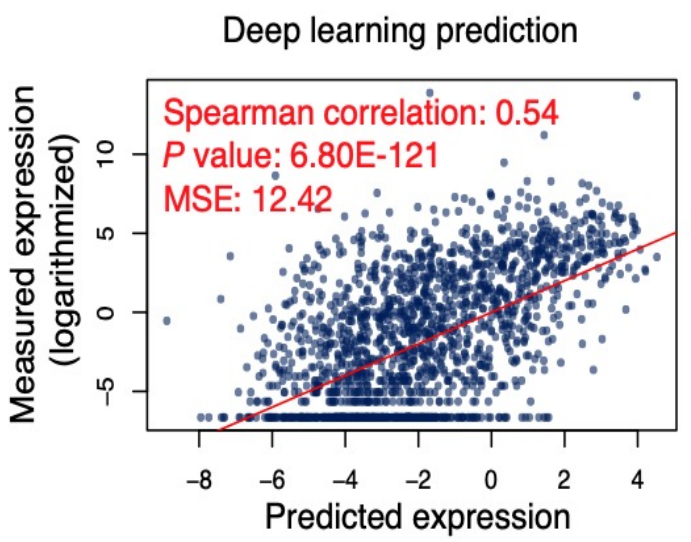

b

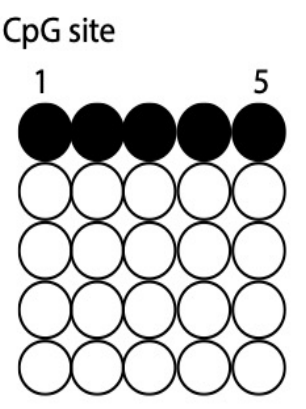

Traditional methylation: 0.2 CHALM: 0.2
C

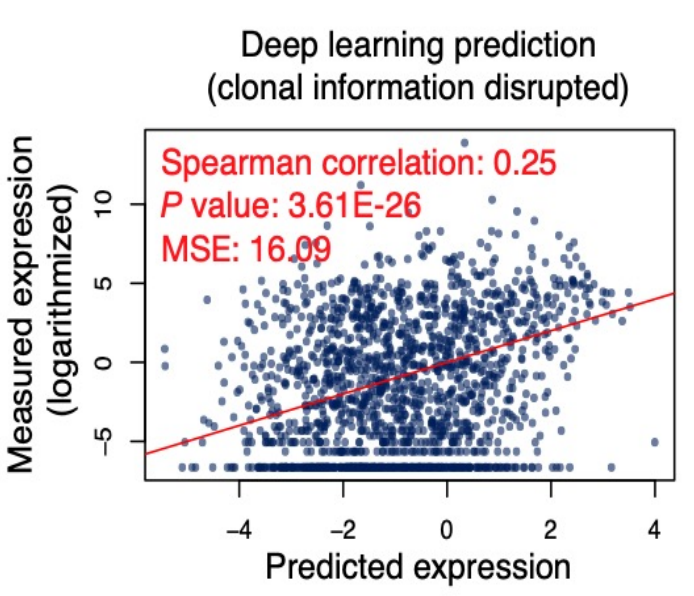

CpG site

Disrupt clonal information by assigning $\mathrm{mCpG}$ to a random read

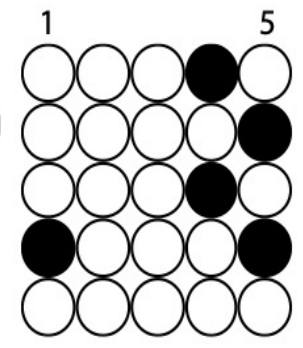

Traditional methylation: 0.2 CHALM: 0.8

Figure 3. Clonal information regarding DNA methylation is important for gene expression prediction. (a) Prediction of gene expression based on raw bisulfite sequencing reads via a deep-learning framework. (b) Disruption of read clonal information by shuffling the $\mathrm{mCpGs}$ among mapped reads. (c) The clonal information is disrupted before prediction. Comparison of correlation (between prediction models with and without clonal information disrupted) permutation $P$ values: $<1 \times 10^{-4}$. 


\section{a}

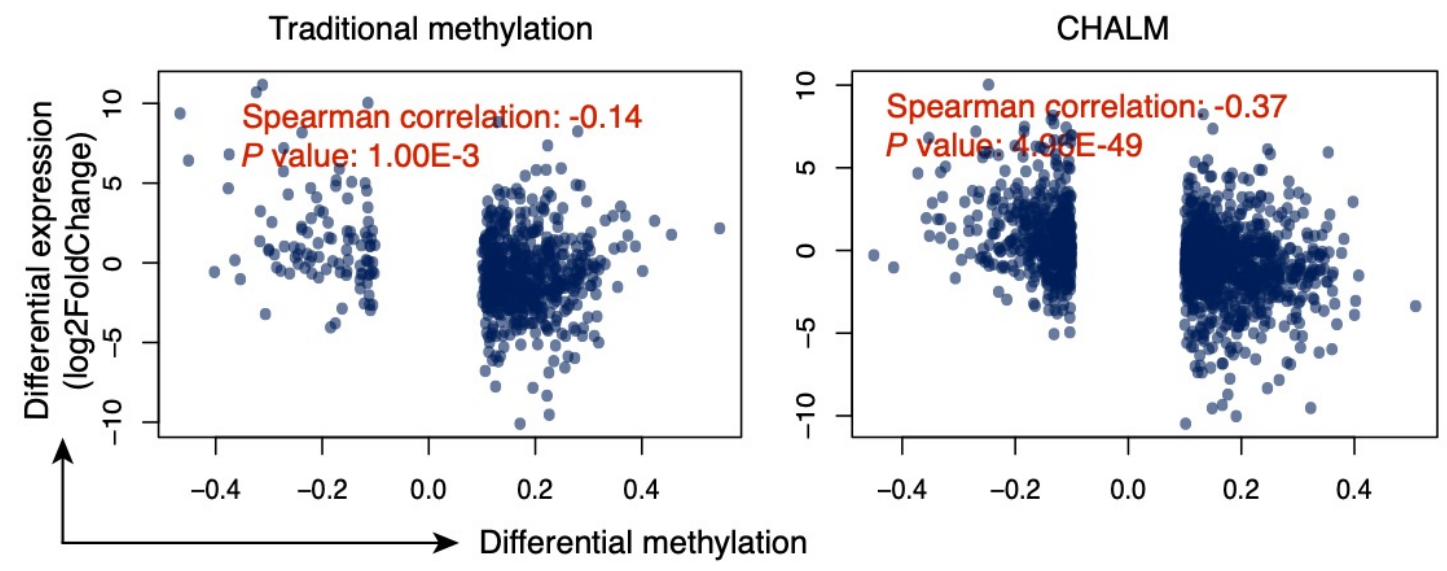

b
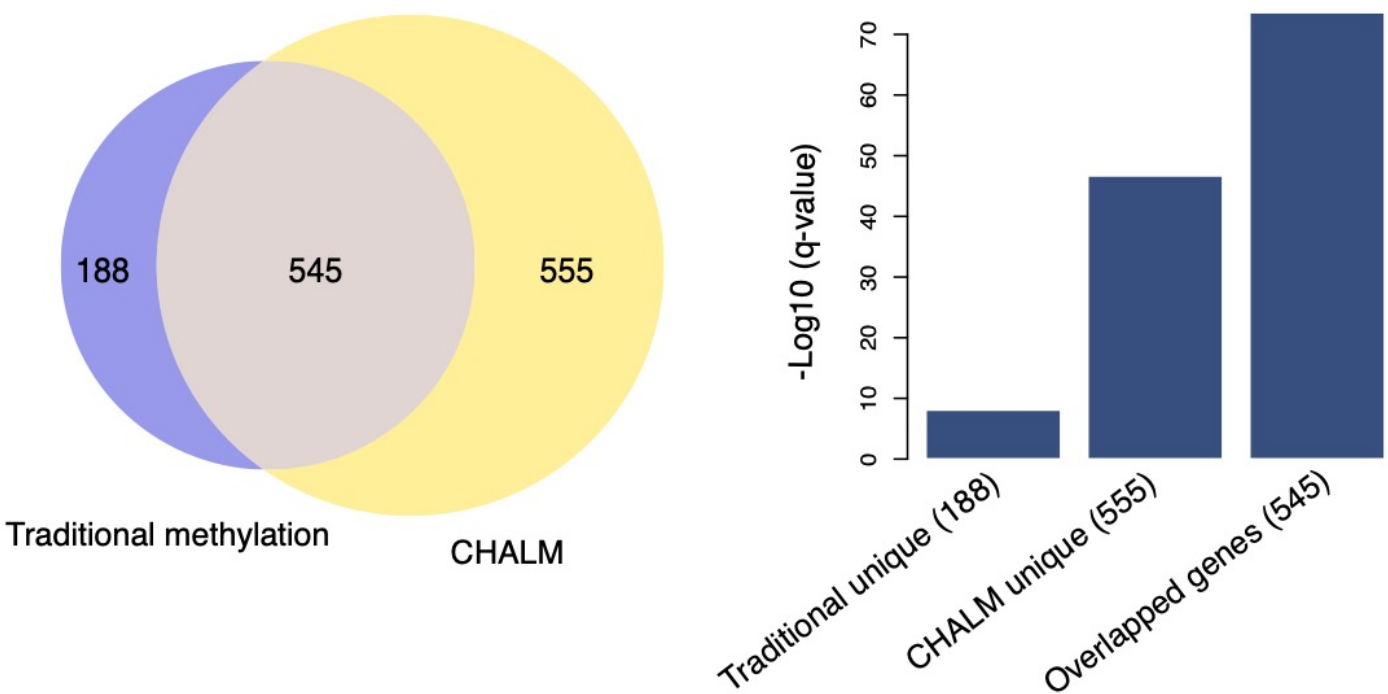

Figure 4. The CHALM methods provides better identification of hypermethylated promoter CGls during tumorigenesis. (a) Scatter plots show the correlation between differential expression and differential methylation calculated by the traditional and CHALM methods. All promoter CGls were included for analysis, but only those exhibiting a significant methylation change between normal and cancerous lung tissue were plotted. X-axis: differential methylation ratio; $y$-axis: differential expression (log2FoldChange). Comparison of correlation (between the traditional method and CHALM) permutation $P$ values: $<1 \times 10^{-4}$. (b) A large fraction of hypermethylated promoter CGls identified by the traditional method can be recovered using the CHALM method, as indicated by the Venn diagram. Bar plot shows enrichment of the H3K27me3 peak in three different gene sets. 


\section{a}

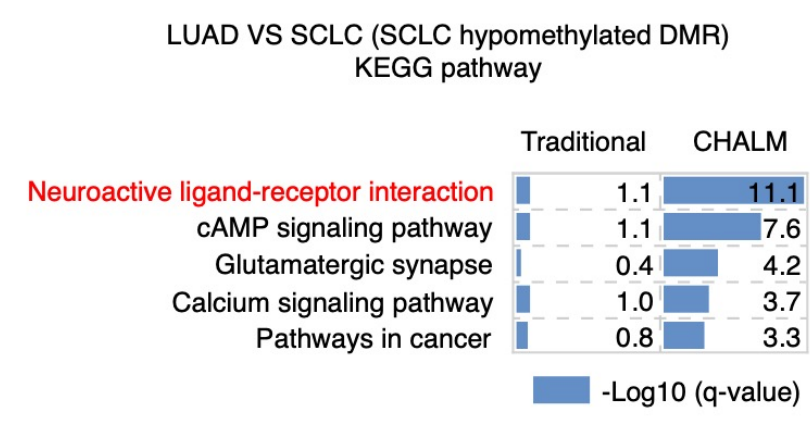

d

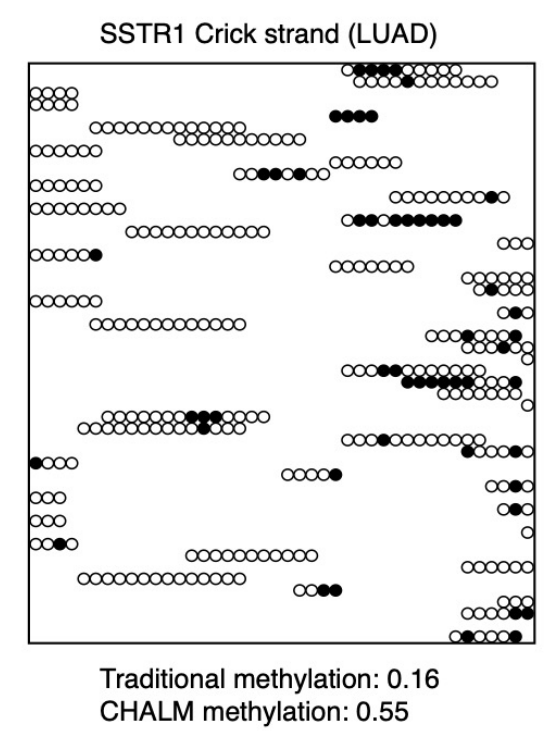

b

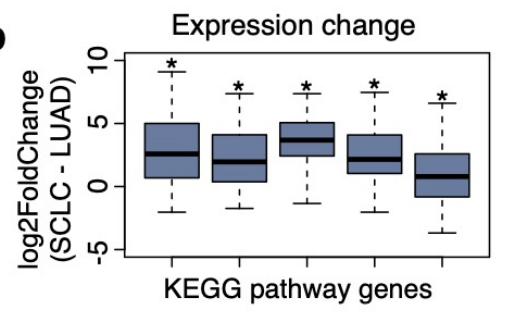

c

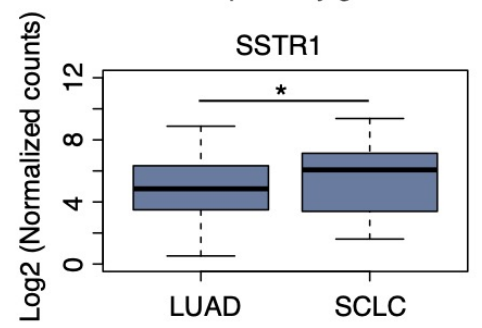

SSTR1 Crick strand (SCLC)

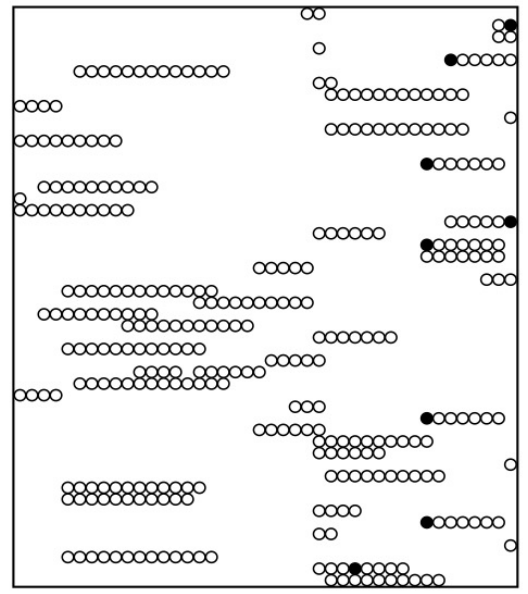

Traditional methylation: 0.02 CHALM methylation: 0.14

Figure 5. The CHALM method provides better identification of functionally related DMRs. (a) KEGG pathway enrichment of the top 2000 hypomethylated DMRs in SCLC. (b) Expression change (SCLC - LUAD) of genes in the KEGG pathways shown. The left-to-right order is the same as the top-to-right order shown in (a). Line in the box center refers to the median, the limits of box refer to the $25^{\text {th }}$ and $75^{\text {th }}$ percentiles and whiskers represent the 1.5 times interquartile range. Two-sided one sample t-test is used. Asterisks indicate a $P$ value of less than 0.05 . (c) Expression of SSTR1 in LUAD (79) and SCLC (79) patients. Two-sided two samples t-test $P$ value, less than 0.05. (d) Methylation status of reads mapped to the CHALM-unique hypomethylated DMR found in the SSTR1 promoter region. Only 50 reads are selected for visualization. The methylation levels shown were calculated based on the original dataset. Black circles: mCpG; white circles: CpG. 